\title{
Disnea en el servicio de urgencia
}

\section{Dyspnea in the emergency deparment}

\author{
María Rosa Sandoval', Bárbara Lara' , Andrés Guzmán ${ }^{1,2}$
}

\section{Resumen}

La disnea es definida como la sensación subjetiva de ahogo o falta de aire y es un motivo muy frecuente de consulta. Su presentación clínica puede variar, desde manifestaciones leves hasta insuficiencia respiratoria catastrófica, con elevada mortalidad y requerir de terapias invasivas complejas. En los servicios de urgencia se inicia el estudio etiológico del paciente agudo, al mismo tiempo que se realizan intervenciones terapéuticas destinadas a la estabilización y manejo tiempo-dependiente del paciente que consulta por disnea. En vista de las múltiples causas de disnea, es necesario que el especialista en medicina de urgencia conozca los distintos diagnósticos diferenciales y sepa orientar su manejo y estudio. Este manuscrito pretende dar un marco teórico acerca de la presentación del paciente con disnea en el servicio de urgencia, describir sus principales características y orientar el estudio y tratamientos tiempo-dependientes desde su primera evaluación por el equipo médico. Se expone un caso clínico y revisan los componentes esenciales de la fisiopatología que explica la disnea, asi como la descripción de herramientas para su evaluación, tratamiento y disposición en el servicio de urgencia. Finalizaremos con la resolución del caso.

Palabras clave: disnea; cansancio; ahogo; disnea cardiogénica; emergencia; urgencia.

\begin{abstract}
:
Dyspnea, defined as the subjective feeling of shortness of breath, it's a common complaint in emergency departments all over the world. The clinical presentation may include mild symptoms to severe respiratory distress with the requirement of mechanical ventilation. This implies high mortality rates and complex decision-making involving diagnostics, treatment, and invasive management in all groups of ages. It is paramount for the emergency medicine physician to acknowledge its vastly differential diagnostics and be familiarized with time-dependent actions to properly stabilization and treatment. This article aims to review the presentation of dyspneic patients in the Emergency Department, describes the main physiologic characteristics, guide diagnosis, and treatment since the very first-minute patient steps into the hospital. It will present clinical scenarios and handle valuable tools for evaluation and treatment strategies in dyspneic patients while they stay in the Emergency Department.
\end{abstract}

Keywords: dyspnea; shortness of breath; cardiogenic dyspnea; emergency department; lung ultrasound.

Fecha de envío: 9 de diciembre de 2019 - Fecha de aprobación: 2 de septiembre de 2020

\section{Caso clínico}

Ingresa al servicio de urgencia una mujer de 45 años de edad con antecedentes de insuficiencia cardíaca (IC), tabáquica crónica, en estudio por enfermedad pulmonar obstructiva crónica (EPOC), obesidad mórbida y diabetes. En la evaluación primaria destaca que habla con frases entrecortadas. Se presenta con una frecuencia respiratoria de 40 por minuto, uso de musculatura accesoria, cianosis peribucal, leve ingurgitación yugular, saturando $84 \%$ ambiental, con sibilancias audibles, crépitos bilaterales a la auscultación. En lo circulatorio: presión arterial (PA) de 175/60 mmHg, frecuencia cardiaca (FC) de 130 latidos por minuto, llene capilar 3 segundos, ritmo regular en 2 tiempos sin soplos, diaforética y fría a distal. Neurológicamente en sopor superficial, pupilas isocóricas reactivas, movilizando las 4 extremidades en forma espontánea. A la exposición con leve edema simétrico de extremidades inferiores,

(1) Hospital clínico Pontificia Universidad Católica de Chile, sección medicina de urgencia, región Metropolitana, Santiago, Chile. (2) Servicio de urgencia hospital Metropolitano Dra. Eloísa Díaz la Florida, Servicio de salud Metropolitano sur oriente, Región Metropolitana, Santiago, Chile.

Autor de Correspondencia: andres.guzman@uc.cl 
HGT $120 \mathrm{mg} / \mathrm{dL}$ y temperatura de $36^{\circ} \mathrm{C}$. Usted decide organizar a su equipo, solicita apoyo de kinesiología y se prepara para manejo de vía aérea avanzada. Se inicia con la monitorización de la paciente, le instala 2 vías venosas periféricas gruesas y le conecta una mascarilla de no recirculación. Solicita un electrocardiograma (ECG) de 12 derivadas. Al respeto discutiremos, al final de entregar los aspectos teóricos, el enfrentamiento inicial de esta paciente

\section{Introducción}

La disnea es definida como la sensación subjetiva de ahogo o falta de aire. Otros términos frecuentemente utilizados para esta sensación son la dificultad respiratoria, fatigabilidad o sed de aire. La dificultad respiratoria, como motivo de consulta primario, es responsable en Estados Unidos de aproximadamente de 4 millones de consultas en el servicio de urgencia (SU) al año (Debos \& Jacobson, 2016). Se presenta más frecuentemente en las edades extremas, principalmente en el período de inverno. En Chile, el año 2016, un 30\% del total de las consultas que se presentaron en los servicios de urgencia (SU) a lo largo de nuestro país fueron por causa respiratoria (MINSAL 2008-2017). Además, la disnea es uno de los síntomas más comunes que requiere de hospitalización desde el SU (Trinquart et al., 2011). Un estudio observacional realizado en Asia mostró que el $10 \%$ de las consultas de urgencia por disnea es admitida para hospitalización. De estas últimas, el 19\% ingresa a una unidad de cuidados intensivos (UCI). Los diagnósticos más frecuentemente reportados fueron insuficiencia respiratoria baja (20\%), insuficiencia cardíaca (IC) (14\%), enfermedad pulmonar obstructiva crónica (EPOC) (13\%) y asma (12\%). El 6\% de los hospitalizados, falleció durante la hospitalización, principalmente por causa de infección respiratoria baja. Kelly et al. (2017) describe que hasta un $15 \%$ se presenta conjuntamente como causa de un componente cardíaco y respiratorio, siendo altamente prevalente en mujeres más que en hombres y por sobre todo en los periodos de embarazo y menopausia, así como también en aquellos pacientes que presentan algún grado de obesidad. Por otro lado, se ha demostrado que presentar disnea sería un predictor independiente de mortalidad en pacientes con IC, EPOC y adultos mayores (Figarska et al., 2012; Mahler \& O’Donnell, 2015).

Por último, la disnea es el síntoma cardinal de una serie de patologías de distinta gravedad y origen, incluyendo anemia, cetoacidosis, así como patologías cardíacas o pulmonares y esconde un espectro de diagnósticos diferenciales relevantes. Es por esto que nos parece de suma importancia exponer las principales herramientas que permiten estratificar el riesgo y encausar la búsqueda diagnóstica del paciente que se presenta con disnea en el contexto de la urgencia.

\section{Generalidades}

Existen en la literatura muchas definiciones para disnea, sin embargo todas concuerdan en que es subjetivo e involucra sensación de falta de aire, dificultad para respirar o sed de aire (Kline et al., 1999; Banzett et al., 2015; Viniol et al, 2015; Barbera et al., 2016). La American Thoracic Society la define como un "término utilizado para caracterizar una experiencia subjetiva de molestias respiratorias que consisten en sensaciones cualitativamente distintas que varían en intensidad" (Meek et al., 1999).

La base fisiopatológica de este síntoma proviene de la interacción de los quimiorreceptores, los receptores de estiramiento, el cerebro y los pulmones, los cuales finalmente modulan la respiración. Los quimiorreceptores detectan los cambios de oxígeno $\left(\mathrm{O}_{2}\right)$ y dióxido de carbono $\left(\mathrm{CO}_{2}\right)$ en la sangre, con lo cual posteriormente activan el impulso respiratorio. La disminución de la ventilación y el aumento del espacio muerto de los pulmones aumentan la presión parcial de $\mathrm{CO}_{2}\left(\mathrm{pCO}_{2}\right)$ en la sangre. El espacio muerto del pulmón crece cuando las unidades pulmonares se ventilan pero no se perfunden, como en el tromboembolismo pulmonar (TEP), mientras que el $\mathrm{CO}_{2}$ aumenta en ciertas patologías como en la enfermedad pulmonar obstructiva crónica (EPOC). Otros quimiorreceptores, son responsables de la detección de acidosis, lo cual también aumenta el impulso respiratorio.

La hipoxemia modula la respiración a través de quimiorreceptores. Cuando el cuerpo carotideo percibe una disminución de oxígeno, estimula al tronco encefálico para aumentar la ventilación. La causa más común de hipoxemia es la incompatibilidad pulmonar ventilación-perfusión. Este desequilibrio entre el flujo sanguíneo pulmonar y ventilación alveolar generalmente se deben a enfermedades del corazón o del pulmón. El shunt es una forma extrema de desajuste de la ventilación-perfusión y ocurre cuando la ventilación a una unidad pulmonar se interrumpe a pesar de la persistencia de flujo sanguíneo; así el paso de sangre evita esta área de pulmón sin intercambiar gases. Durante el shunt, el cuerpo compensa con vasoconstricción pulmonar hipóxica o refleja, que atenúa el flujo sanguíneo a aquel pulmón con unidades no ventiladas. Mientras que los agentes beta 2-adrenérgicos pueden revertir esta respuesta vascular, el oxígeno suplementario no puede corregir la hipoxemia producida por el shunt.

La resistencia elevada de la vía aérea, el espacio muerto elevado y la rigidez pulmonar anormal, pueden aumentar el trabajo respiratorio. Los mecanorreceptores de la cara, vías respiratorias superiores, pared torácica, son responsables para la retroalimentación que modula esta sensación en donde los receptores J vagales en el 
pulmón son mediadores importantes. Investigadores creen que la discrepancia entre volumen pulmonar y tensión de los músculos de la respiración, es otro factor importante con incremento del trabajo respiratorio.

Incluso la disnea psicógena tiene una base fisiopatológica, donde cambios en la neuroquímica cerebral y una inusual respuesta a la presión parcial de $\mathrm{CO}_{2}\left(\mathrm{pCO}_{2}\right)$, tal vez sea responsable de la falta de aire o trastorno de pánico (Kline et al., 1999; Meek et al., 1999)

Si bien no es fácil ordenar las etiologías causantes de disnea, hacer una consideración anatómica o por sistemas resulta más fácil de recordar. Estos incluyen vía aérea, pulmón, corazón, hematológico/ metabólico y causas neuromusculares. Recordar que la debilidad muscular puede producir disnea y sus causas incluyen miastenia gravis, síndrome Guillain-Barré y tirotoxicosis. El reflujo gastroesofágico es responsable de $4 \%$ disnea crónica indiferenciada (Meek et al., 1999; Viniol et al., 2015; Debos \& Jacobson, 2016). En la tabla 1 se enumeran las principales causas.

Tabla 1: Causas comunes de disnea.

\begin{tabular}{|c|c|}
\hline $\begin{array}{l}\text { Vía aérea alta } \\
\text { Cuerpo extraño } \\
\text { Reacción alérgica } \\
\text { Masa } \\
\text { Estenosis via aérea } \\
\text { Traqueomalacia }\end{array}$ & $\begin{array}{c}\text { Metabólica/Hematológica } \\
\text { Tirotoxicosis } \\
\text { Hemoglobinas anormales (Monoxi- } \\
\text { do de carbono/metahemoglobina) } \\
\text { Anemia } \\
\text { Desorden de fosfato, potasio o } \\
\text { calcio } \\
\text { Sepsis/Fiebre } \\
\text { Acidosis }\end{array}$ \\
\hline $\begin{array}{c}\text { Pulmón/Vía aérea baja } \\
\text { Neumonía } \\
\text { Neumotórax } \\
\text { Derrame pleural } \\
\text { Tromboembolismo pulmonar } \\
\text { Hipertensión pulmonar } \\
\text { Enfermedad pulmonar intersticial } \\
\text { Síndrome distres respiratorio } \\
\text { del adulto } \\
\text { Enfermedad pulmonar } \\
\text { obstructiva crónica } \\
\text { Asma } \\
\text { Tumores }\end{array}$ & $\begin{array}{l}\text { Neuromuscular } \\
\text { Guillain-Barré } \\
\text { Miastenia gravis } \\
\text { Miopatía } \\
\text { Neuropatía }\end{array}$ \\
\hline $\begin{array}{c}\text { Cardíaco } \\
\text { Isquemia miocárdica } \\
\text { Insuficiencia cardíaca congestiva } \\
\text { Derrame pericardico } \\
\text { Valvulopatias } \\
\text { Arritmias }\end{array}$ & $\begin{array}{c}\text { Psicogénico/otras } \\
\text { Desorden de pánico } \\
\text { Hiperventilación } \\
\text { Ascitis masiva } \\
\text { Síndrome abstinencia a drogas }\end{array}$ \\
\hline
\end{tabular}

\section{Diagnósticos diferenciales}

La diversidad de condiciones fisiológicas que producen disnea hace que el enfrentamiento muchas veces sea difícil. Si bien, el estudio acabado debe considerar todos los sistemas antes mencionados, en el servicio de urgencia existen diagnósticos críticos que deben ser considerados rápidamente para determinar las mejores opciones de tratamiento para estabilizar el paciente (Braithwaite \& Perina, 2013). En la tabla 2, se muestran los diagnósticos diferenciales para la disnea aguda, esta vez ordenados de acuerdo a su gravedad.

Tabla 2: Enfrentamiento de los diagnósticos diferenciales de disnea aguda.

\begin{tabular}{|c|c|c|}
\hline $\begin{array}{c}\text { Diagnósticos } \\
\text { críticos }\end{array}$ & $\begin{array}{c}\text { Diagnósticos } \\
\text { emergentes }\end{array}$ & $\begin{array}{c}\text { Diagnósticos no } \\
\text { urgentes }\end{array}$ \\
\hline $\begin{array}{c}\text { Obstrucción de vía } \\
\text { aérea }\end{array}$ & $\begin{array}{c}\text { Neumotórax espon- } \\
\text { TEP }\end{array}$ & $\begin{array}{c}\text { Déneo } \\
\text { Edema pulmonar }\end{array}$ \\
Anafilaxia & Asma & Neoplasia \\
Insuficiencia respira- & Neumonía & EPOC \\
toria & Pericarditis & IC \\
IAM & Sepsis & Valvulopatía \\
Taponamiento & Falla renal & Miocardiopatía \\
cardíaco & Anormalidades & Embarazo \\
Cetoacidosis & Ascitis \\
Epiglotitis & ácido-base & Obesidad \\
Neumotórax a tensión & Ruptura & Hiperventilación \\
Tórax volante & diafragmática & Pánico \\
Intoxicación por mo- & Anemia & Fiebre \\
nóxido de carbono & Esclerosis múltiple & Enfermedad de la \\
Intoxicación por orga- & Síndrome Guil- & tiroides \\
nofosforados & lain-Barré & Fractura costal \\
Derrame pleural & & Polimiositis \\
masivo & & \\
Hemotórax & & \\
\hline
\end{tabular}

TEP:Tromboembolismo pulmonar. Infarto agudo al miocardio: IAM. EPOC: Enfermedad pulmonar obstructiva crónica. IC: Insuficiencia cardiaca.

La frecuencia de estos diagnósticos es distinto dependiendo del lugar donde se esté atendiendo el paciente. En los servicios de rescate extrahospitalarios predominan la insuficiencia cardíaca (15-16\%) y la neumonía (10-18\%). Le siguen la enfermedad pulmonar obstructiva crónica con un $13 \%$, y el asma con un $5-6 \%$. Otras causas cardiovasculares como el Infarto agudo al miocardio y el tromboembolismo pulmonar que son los responsables de un $4 \%$ y un $2 \%$ respectivamente. El cáncer de pulmón sólo es responsable del $1 \%$ de las consultas por disnea en los servicios de rescate extrahospitalario. En servicios de urgencia hospitalarios, los diagnósticos más comunes son la enfermedad pulmonar obstructiva crónica (17\%), la insuficiencia cardíaca (16\%), neumonía (8\%), el Infarto agudo al miocardio (5\%), fibrilación auricular (FA) o flutter con respuesta ventricular rápida (4\%), tumor maligno (3\%) y el tromboembolismo pulmonar (3\%) (Berliner et al., 2016).

Otra manera de enfocar los diagnósticos al momento de enfrentarse a un paciente con disnea es hablar de disnea obstructiva, parenquimatosa, circulatoria y compensatoria (Debos \& Jacobson, 2016).

En la disnea por causa obstructiva, típicamente encontraremos ruidos agregados como sibilancias, los que resultan de obstrucción 
parcial de la vía aérea alta (supraglótica) o baja (árbol bronquial). Esta obstrucción puede ser secundaria a secreciones o incluso un cuerpo extraño. Frecuentemente las sibilancias son causadas por alteraciones de la vía aérea baja y el estridor es sonido de la vía aérea alta. Las causas van desde anafilaxia, exacerbación asmática o enfermedad pulmonar obstructiva crónica.

En la disnea por causa parenquimatosa, se produce alteración en la difusión de gases dado por patologías que causan relleno alveolar o aumento del espacio intesticial pulmonar. Por ejemplo, la congestión intersticial por falla cardiaca aguda o por otro lado, el infiltrado alveolar inflamatorio por una neumonía, presenta una limitación de la difusión de oxígeno, lo que finalmente lleva a falta de aire. En relación a este último, se sabe que los criterios clínicos de tos, fiebre, esputo y disnea, tienen una sensibilidad del $50 \%$ comparado con la radiografía de tórax. Por lo que la American Thoracic Society (ATS) recomienda que en los pacientes que se sospeche neumonía, se debiese tomar una radiografía de tórax. Hay otras neumonías importantes de mencionar, las cuales están asociadas a la atención de salud. Por definición, en esta categoría entran todos los pacientes que presentan síntomas 48 horas después del ingreso al hospital o después de 48-72 horas post-intubación endotraqueal; y esta se puede presentar hasta 90 días post-alta del paciente. Esta definición temporal es importante ya que el tratamiento antibiótico se enfoca a germenes distintos que la neumonía adquirida en la comunidad.

Otro tipo de disnea es la circulatoria, y aquí el tromboembolismo pulmonar es una importante causa a considera en nuestros diferenciales, en donde la interferencia de la ventilación/perfusión (V/Q) es un gran problema, lo cual finalmente termina en colapso circulatorio debido a sobrecarga del ventrículo derecho (VD) por aumento de presión de la arteria pulmonar. Nosotros podemos encontrar ciertos hallazgos ecocardiográficos, tales como dilatación del VD, hipocinesia del VD o signo de McConnell (hipocinesia de la pared libre del VD e hiperdinamia del ápex) y regurgitación tricuspidea, que nos va a orientar en el diagnóstico.

Por otro lado, las reglas predictivas también nos brindan ayuda. Una herramienta ampliamente conocida y útil es el score de Wells que se puede aplicar frente a un paciente con disnea y/o dolor torácico (Wells et al., 2001). A su vez, en un paciente en donde nuestra sospecha de TEP es baja, la regla predictiva PERC (Pulmonary Emolism Rule-out Criteria) nos permite ser más costo-efectivos, siendo superior a score de Wells en este grupo de pacientes. Por su parte, el dímero $D$, también nos aporta en el diagnóstico de trombosis pulmonar, siendo su mayor utilidad su valor predictivo negativo cercano a $100 \%$. Finalmente si nuestra sospecha es alta, es mandatoria la realización de un angio-TAC de tórax para la confirmación diagnóstica.
Otro diagnóstico a considerar en este grupo es el infarto agudo al miocardio, en donde se debe considerar el historial médico y los factores de riesgo al momento de la sospecha diagnóstica. En ellos, resulta perentoria la realización de un electrocardiograma (ECG), enzimas cardíacas si amerita y radiografía de tórax para su diagnóstico y estratificación; así como también evaluar los otros diagnósticos diferenciales de dolor torácico y disnea.

El neumotórax se debe considerar en aquellos pacientes altos, delgados con tabaquismo en donde puede haber riesgo de rotura por daño de la capa pleural. El diagnóstico en este caso puede ser realizado por ultrasonografía, radiografía de tórax y escáner de tórax. En aquellos pacientes con neumotórax a tensión la descompresión inmediata es necesaria.

Finalmente la disnea compensatoria, incluye aquellas patologías tales como anemia, acidosis metabólica (por ejemplo, en cetoacidosis diabética) o dolor. Existen casos también donde la disnea es un diagnóstico de exclusión, en donde la ansiedad aguda, los trastornos de pánico y la hiperventilación se pueden presentar como dificultad respiratoria (Kline et al., 1999; Braithwaite \& Perina, 2013; Berliner et al., 2016; Debos \& Jacobson, 2016).

\section{Enfrentamiento inicial}

En el enfrentamiento y evaluación inicial es importante definir si el paciente requiere una intervención inmediata con ayuda de la evaluación de su apariencia inicial, signos vitales y evaluación primaria estructurada (Barbera et al., 2016). Uno de los principales objetivos de la medicina de urgencia es la detección oportuna de causas graves o potencialmente mortales de disnea, por lo que la disnea psicogénica debe ser diagnosticada después de excluir causas orgánicas. Existe evidencia que indica que con la historia, el examen físico y la radiografía de tórax, se puede identificar la causa de disnea en hasta 2/3 de los pacientes (Kline et al., 1999; Meek et al., 1999). En la tabla 3 se enumeran los hallazgos clínicos y su correlación con el diagnóstico.

Tabla 3: Hallazgos clínicos y su correlación diagnóstica.

\begin{tabular}{l|l}
\hline Síntoma o signo & Diagnóstico diferencial \\
\hline Jadeo & EPOC/Enfisema, asma, reacción alérgica, IC \\
\hline Tos & Neumonía, asma, EPOC/enfisema \\
\hline Dolor pleurítico & Neumonía, TEP, neumotórax, EPOC, asma \\
\hline Ortopnea & IC \\
\hline Fiebre & Neumonía, bronquitis, TBC, tumor pulmonar \\
\hline Hemoptisis & Neumonía, TBC, TEP, tumor pulmonar \\
\hline Edema & IC/EPA, TEP (unilateral) \\
\hline Congestión pulmonar & ICaguda o crónica/EPA, ERCoDHC, SDRA (por sepsis) \\
\hline Taquipnea & $\begin{array}{l}\text { TEP, acidosis (incluyendo toxicidad por aspirina), } \\
\text { ansiedad }\end{array}$ \\
\hline
\end{tabular}

EPOC: Enfermedad pulmonar obstructiva crónica. IC: Insuficiencia cardiaca. TEP: Tromboembolismo pulmonar. TBC: Tuberculosis. EPA: Edema agudo de pulmón. ERC: Enfermedad renal crónica. DHC: daño hepático crónico. SDRA: sindrome de distres respiratorio. 
La anamnesis resulta clave en buscar síntomas acompañantes, como diaforesis, dolor torácico, palpitaciones, tos o fiebre. Estos síntomas proporcionan pistas importantes que orientan a la etiología. La edad, los antecedentes médicos y los síntomas asociados nos orientan en la estratificación de riesgo. El tiempo de evolución, la duración y progresión de los síntomas proporcionan información clave de la historia natural de la enfermedad y la gravedad potencial de esta. La observación de factores desencadenantes puede proporcionar información etiológica y conducir al médico hacia la modalidad de tratamiento más adecuada. Si se dispone de tiempo, se debe profundizar en claves para el diagnóstico etiológico específico; saber la ocupación por exposición laboral a ciertos agentes o enfermedades laborales, si la disnea se desencadena en ejercicio o no, asociación con ortopnea o disnea paroxística nocturna, edema de extremidades inferiores, tabaquismo, trauma, viajes, sangrados, uso de medicamentos, entre otros (Kline et al., 1999; Barbera et al., 2016; Debos \& Jacobson, 2016).

Con respecto al examen físico, hay que poner atención principalmente al sistema cardiovascular y pulmonar. Los signos vitales y el trabajo respiratorio son parte de la evaluación inicial, con énfasis en la permeabilidad de las vías respiratorias (como movilización de secreciones, presencia de edema laríngeo u otros), frecuencia respiratoria, capacidad de hablar en frases completas, cambio de voz o ruido respiratorio audible y uso de musculatura accesoria.

Una vez completa la evaluación primaria se deben realizar las intervenciones inmediatas destinadas a corregir las alteraciones encontradas. La auscultación de los sonidos cardíacos (R3, galope, soplos, etc.) y respiratorios (sibilancias, crépitos, roncus, entre otros) proporcionan pistas a la fisiología subyacente de la enfermedad. Se debe completar la evaluación examinando el abdomen en búsqueda de reflujo hepatoyugular en la insuficiencia cardiaca por ejemplo; la presencia de edema bilateral o unilateral en las extremidades y buscar rash o enfisema subcutáneo en piel. La combinación de hipotensión y disnea es ominoso, las etiologías que causan esta condición son de mal pronóstico e incluyen condiciones tales como tromboembolismo pulmonar, shock, neumotórax, hemorragias ocultas y desórdenes metabólicos (Kline et al., 1999; Barbera et al., 2016; Debos \& Jacobson, 2016).

Existen diversas escalas de grado de severidad de disnea, una de ellas es la escala de Borg. En esta escala, se le pide al paciente que califique su disnea de 0 a 10. Cero es sin dificultad respiratoria y diez es severo con síntomas opresivos. En la tabla 4, se muestra la descripción de esta escala.
Tabla 4: Escala de Borg.

0 Nada en lo absoluto
1 notable leve
2 muy leve
3 ligero
4 ligero - moderado
5 moderado
6 alguna dificultad
7 moderadamente severa
8 severa
9 muy severa
10 nivel pánico, máxima disnea

\section{Estudio}

El electrocardiograma (ECG) es una herramienta útil dentro de nuestras acciones primarias en la evaluación del paciente con disnea, ya que nos brinda información en el caso de cursar arritmias, IAM o signos de enfermedades cardiopulmonares tales como, tromboembolismo pulmonar, pericarditis o hipertensión pulmonar. A favor de este test está el hecho que es rápido, de bajo costo y al lado de la cama del paciente. Un electrocardiograma (ECG) normal hace que la insuficiencia cardíaca sea un diagnóstico poco probable en un paciente con disnea, ya que se sabe que un ECG normal tiene un valor predictivo negativo (VPN) de 98\% para la disfunción sistólica del ventrículo izquierdo. Por el contrario, encontrar ondas T negativas y simétricas en las derivadas V1-V4 es 85\% sensible y $81 \%$ específico para el diagnóstico de tromboembolismo pulmonar de riesgo moderado y alto. Por su parte, el signo S1Q3T3 o complejo de McGinn-White, tiene una sensibilidad de sólo 54\% y especificidad de $62 \%$, por lo que no nos permite ni confirmar ni descartar el diagnóstico (Kline et al., 1999; Barbera et al., 2016; Debos \& Jacobson, 2016).

La imagenología proporciona un apoyo invaluable en el estudio de los pacientes con disnea en el servicio de urgencia.

La radiografía de tórax es útil como primer test diagnóstico en un paciente indiferenciado. Nos muestra el tamaño de la silueta cardíaca, mediastino, silueta aortica, infiltrado pulmonar, la presencia de masas, neumotórax o derrame pleural, anomalías óseas y algunas otras alteraciones (Barbera et al., 2016). Tiene como beneficio la baja irradiación, pero en general tiene baja sensibilidad para la mayoría de los diagnósticos. En pacientes que ingresan clinicamente deshidratados por ejemplo, puede no mostrarse una condensación en una radiografía de tórax muy precoz, por lo que se ha recomendado volemizar empíricamente y realizar un control 
radiográfico posterior (Debos \& Jacobson, 2016). Este método sería de utilidad en pacientes con problemas cardio-rrespiratorios pues muestra anomalías nuevas significativas en más de un tercio de los pacientes (Kline et al., 1999).

Por su parte, la tomografía computada de tórax ofrece buena sensibilidad y especificidad para el diagnóstico de varias patologías respiratorias. Sin embargo, es un examen costoso, involucra irradiación y exposición a efectos adversos del medio de contraste. En el caso del estudio del TEP, el colegio Americano de médicos radiólogos recomienda no realizarlo de rutina en todos los pacientes con disnea que tengan bajo riesgo de presentar la enfermedad (Debos \& Jacobson, 2016).

El ultrasonido es otra herramienta que tiene varios beneficios. Por un lado, carece de radiación ionizante, es rápido, se puede realizar al lado de la cama del paciente y proporciona información valiosa sobre el origen de los síntomas. A menudo, nos permite orientar el diagnóstico precozmente en el paciente con disnea aguda, lo cual además guiará en la toma de decisiones y la opción a una terapia oportuna. El protocolo más conocido es el "Bedside Lung Ultrasonography in Emergency (BLUE)", creado en el año 2008 y nos permite diferenciar las distintas causas de insuficiencia respiratoria. El examen de pulmón, permite pesquisar la presencia de neumotórax si es que hay ausencia de deslizamiento pleural o el hallazgo del punto pulmonar tiene un valor predictivo positivo (VPN) de 100\% para el diagnóstico de neumotórax. En el caso de sospechar congestión pulmonar, basándose en la presencia de líneas B simétricas (Figura 1) en todos los cuadrantes pulmonares evaluados, se hace el diagnóstico de edema intersticial pulmonar, con una sensibilidad de $85,7 \%$ y especificidad de $97 \%$. Se plantea que la precisión diagnóstica de esta prueba llegaría a ser cercana al 90,5\%, además de reducir los tiempos en descifrar el diagnóstico, también disminuiría el retraso en el manejo de los pacientes (Barbera et al., 2016; Debos \& Jacobson, 2016; Seyedhosseini et al., 2017; Wimalasena et al., 2017; Zanobetti et al., 2017).

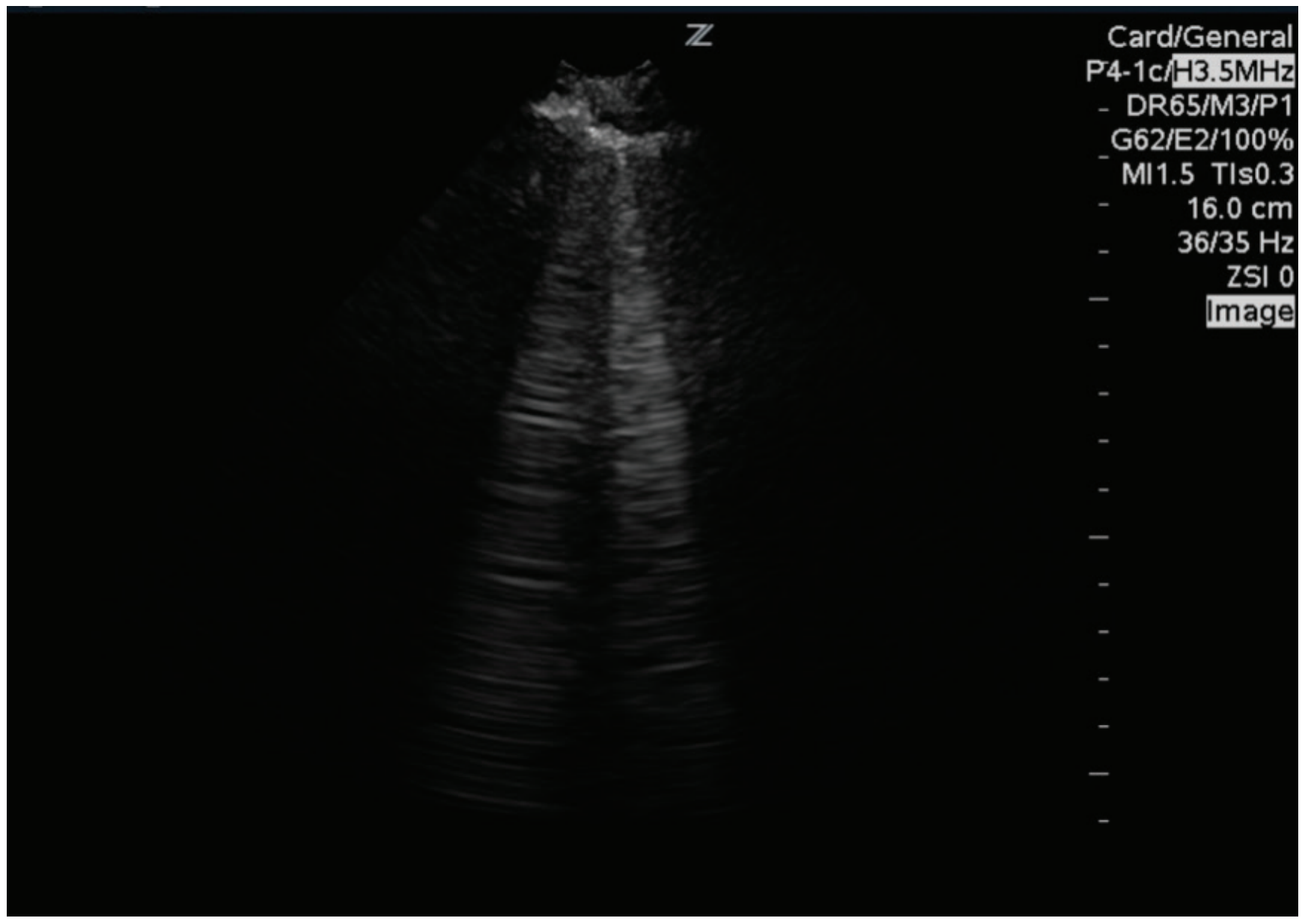

Figura 1: Líneas B pulmonares. Lineas hiperecoicas (blancas tipo "cometa") que llegan al final de la pantalla. Traducen edema intesticial pulmonar. 
Otros protocolos ultrasonográficos incluyen la búsqueda de causa cardíaca de disnea, en donde la evaluación focalizada de la función ventricular izquierda global, la función diastólica, el tamaño ventricular derecho y la presencia de derrame pericárdico facilitan la evaluación rápida, sin la necesidad de sacar a nuestro paciente del servicio de urgencia, de diagnósticos como el infarto miocárdico masivo, embolía pulmonar masiva y el taponamiento cardíaco, respectivamente. Se pueden utilizar otros elementos tales como la medición de la vena cava inferior ( $\mathrm{VCl}$ ) y su fluctuación con los movimientos ventilatorios del paciente. Su utilidad radicaría en la evaluación de signos de insuficiencia cardíaca derecha y estimación la presión venosa central (PVC), idea la que actualmente está en cuestionamiento (Barbera et al., 2016; Debos \& Jacobson, 2016).

Con el avance del ultrasonido y el beneficio demostrado en el enfrentamiento de los pacientes con disnea, se han realizado estudios comparando la capacidad diagnóstica de la ecografía al lado de la cama del paciente versus el estetoscopio. Se ha visto que el ultrasonido es más preciso en realizar los diagnósticos de insuficiencia cardíaca y neumonía (Özkan et al., 2015; Zanobetti et al., 2017), En el caso de consolidación por neumonía, se ha demostrado que el ultrasonido tiene una sensibilidad de $89 \%$ y una especificidad de $94 \%$ para el diagnóstico en manos entrenadas (Wimalasena et al., 2017).

Por último, el ultrasonido nos permite rápidamente evaluar la presencia de derrame pleural, con una sensibilidad por sobre el $90 \%$, teniendo incluso mayor sensibilidad que el examen físico y la radiografía de tórax portátil, ya que detecta hasta $20 \mathrm{~mL}$ de fluidos en manos bien entrenadas (Martino et al., 2014; Wimalasena et al., 2017)

El estudio del paciente con disnea debe ser completado con exámenes de laboratorio atingentes a los hallazgos clínicos e imagenológicos y sin duda pueden ayudar en la evaluación y el tratamiento. Un estudio metabólico básico inicial debería incluir alteraciones electrolíticas, función renal y niveles de glucosa. La gasometría arterial o venosa brinda información útil sobre el estado ácido base, oxigenación y ventilación del paciente. Un recuento hematológico nos proporciona información respecto a la presencia de anemia, hemoconcentración y leucocitosis como signo inespecífico de infección. De los marcadores cardíacos, nos interesa la troponina, el cual es marcador específico de daño miocárdico. Sin embargo, se debe tener en cuenta que etiologías como el tromboembolismo pulmonar y la miopericarditis pueden elevarla, por lo que no es un examen específico de infarto agudo al miocardio. La creatinkinasa total (CK-T) y su fracción miocárdica (CK-MB), también son marcadores de daño miocárdico menos específicos, ya que también se alteran en casos de daño de otros órganos sistémicos y pudiesen elevarse frente a la rotura muscular, como en el caso de rabdomiolisis secundario a trauma por ejemplo. El dímero $D$, es un marcador de actividad fibrinolítica, se usa como marcador de trombosis venosa profunda y embolia pulmonar en pacientes de bajo riesgo. Su valor de corte para ser considerado positivo es cuando supera los $500 \mathrm{ng} / \mathrm{mL}$. Su sensibilidad y especificidad para tromboembolismo pulmonar son $98 \%$ y $43 \%$ respectivamente. En un paciente de bajo riesgo de embolía pulmonar y un dímero $D$ negativo se reduce significativamente la probabilidad diagnóstica con un valor predictivo negativo (VPN) 97\%. Es importante considerar que debe realizarse el ajuste del Dímero D por la edad, es decir, sobre los 50 años, se considera positivo si su valor es superior a multiplicar por 10 la edad del paciente. Esta apreciación es para mantener su sensibilidad. Su especificidad se ve limitada por patologías tales como coagulopatía de consumo, infección, malignidad, trauma, disección arterial, embarazo y/o preeclampsia, entre otros.

El péptido natriurético cerebral (BNP) y proBNP es una neurohormona cardiaca que se libera por sobrecarga del miocito, por lo tanto, generalmente se encuentra elevado en insuficiencia cardiaca. Dado lo anterior, su elevación, en contexto de un paciente disneico, sería altamente sugerente de una causa cardiogénica. Hay estudios que han tratado de evaluar la utilidad del proBNP y BNP en pacientes con disnea como predictor de necesidad de hospitalización, días de estadía hospitalaria y mortalidad. Sin embargo, hasta ahora se sabe que podría disminuir sólo en un día la hospitalización y posiblemente reducir la tasa de hospitalización sin un efecto concluyente en la tasa de mortalidad. Tampoco se ha visto beneficio en la probabilidad de rehospitalización. La sociedad europea de cardiología recomienda valores de umbral BNP $<100$ $\mathrm{pg} / \mathrm{mL}$ y para el pro BNP $<300 \mathrm{pg} / \mathrm{mL}$ para descartar insuficiencia cardiaca congestiva, con un valor predictivo negativo (VPN) 94 a 98\%. Importante es considerar que su interpretación se ve limitada en pacientes obesos o con insuficiencia renal. (Kline et al., 1999; Lam et al., 2010; Trinquart et al., 2011; Barbera et al., 2016; Berliner et al., 2016; Debos \& Jacobson, 2016).

\section{Manejo en servicio de urgencia}

Inicialmente debemos posicionar nuestro paciente en sedestación para favorecer la oxigenación y ventilación de las regiones dependientes. El oxígeno suplementario debiera administrarse a todo paciente que consulte por disnea y que esté clínicamente hipoxémico, esto es con una saturación $<90 \%$. Afortunadamente, existen diversos dispositivos de administración y titulación de oxígeno como la cánula nasal, mascarilla de alto flujo, mascarilla de no recirculación, cánula nasal de alto flujo (CNAF), ventilación no invasiva e invasiva. Independiente del dispositivo que elijamos, 
el objetivo es lograr una saturación de oxígeno mayor a 92-94\%. En los pacientes con patología crónica, del tipo limitación crónica del flujo aéreo (LCFA), lograr 88-92\% es suficiente (Kline et al., 1999; Barbera et al., 2016).

La ventilación mecánica no invasiva (VMNI), es una modalidad de asistencia ventilatoria que no requiere de una intubación endotraqueal, siendo esta su principal ventaja. Existen principalmente 2 modos ventilatorios, el CPAP (Continuous Positive Airway Pressure) o ventilación continua a presión positiva, que entrega un nivel de presión de soporte continuo tanto en inspiración como espiración y BiPAP (Bilevel Positive Airway Pressure) o ventilación continua a presión positiva de dos niveles, que entrega dos niveles diferentes de presión tanto en inspiración y espiración, teóricamente más fisiológico y mejor tolerado por los pacientes. Las indicaciones y contraindicaciones del uso de la ventilación mecánica no invasiva (VMNI), se orden en la Tabla 5. Sin embargo, cabe señalar que, en el edema pulmonar agudo, la enfermedad pulmonar obstructiva crónica, la neumonía en inmunosuprimido, la Miastenia gravis y Esclerosis Lateral Amiotrófica (ELA) son parte del grupo de patologías en donde esta modalidad ventilatoria tiene beneficios claros en mortalidad, disminución de intubación e ingreso a unidad de cuidados intensivos (UCl) (Rochwerg et al., 2017).

Tabla 5: Indicaciones y contraindicaciones de VMNI.

Indicaciones
Distrés Respiratorio
EPA
EPOC
Asma
Neumonía en inmunosuprimido
Destete de ventilación mecánica
Alteraciones Metabólicas
Contraindicaciones (absolutas y relativas)
Imposibilidad de protección de vía aérea (compromiso de conciencia,
aumento de secreciones, trauma facial)
Hipotensión
Neumotórax
PCR

EPA: Edema pulmonar agudo. EPOC: Enfermedad pulmonar obstructiva crónica. PCR: Paro cardiorrespiratorio.

La intubación orotraqueal (IOT) se debe usar en casos en donde se requiera la protección de vía aérea por inminente colapso y consecuente hipoxia, por ejemplo edema de vía aérea en la anafilaxia, mal manejo de secreciones en el caso de compromiso de conciencia por patología neurológica, tóxico-metabólica o por falla respiratoria aguda que requiera estrategias ventilatorias con alto nivel de asistencia. Las indicaciones absolutas de intubación orotraqueal se enumeran en la Tabla 6.
Tabla 6: Indicaciones de intubación orotraqueal (IOT)

Falla respiratoria aguda
PCR
Falla VMNI
Imposibilidad de mantener vía aérea permeable
Pérdida inminente de vía aérea (anafilaxia, hipersecreciones, trauma
de vía aérea)
Compromiso de conciencia severo
Riesgo de aspiración
Hipercapnia con acidosis no corregible con VMNI

PCR: Paro cardiorrespiratorio. VMNI: Ventilación mecánica no invasiva.

\section{Disposición del paciente}

Todos aquellos pacientes inestables o con diagnósticos críticos deben ser estabilizados y requieren admisión hospitalaria a unidades de pacientes críticos (UPC). Por su parte, los pacientes que cursan con diagnósticos emergentes que mejoran su condición en la urgencia, pueden ser admitidos en unidades de cuidados intermedios (UTI). Todos aquellos pacientes con diagnósticos no urgentes pero con condiciones de peligro de deterioro, sin opción a tratamiento adecuado ambulatorio, con comorbilidades; tales como diabetes, inmunosupresión o cáncer, pueden requerir hospitalización para observación, más estudio o tratamiento intrahospitario. La mayoría de los pacientes con diagnósticos no urgentes pueden ser tratados en forma ambulatoria con un buen seguimiento médico posterior. Ahora bien, si el paciente persiste con disnea a pesar del tratamiento, reconsulta y no hay alguna causa clara, la mejor opción es hospitalizarlo. Por otro lado, si los síntomas han mejorado, el paciente está estable, se podría dar alta y seguimiento ambulatorio precoz, con signos de alarma bien claros.

\section{Resolución del caso}

Usted decide tomar exámenes de laboratorio, nebulizar con berodual y salbutamol intercalados, le indica corticoides endovenosos. La paciente no responde y evoluciona con mala mecánica ventilatoria, por lo que finalmente se decide conectar a ventilación mecánica no invasiva (VMNI) en modo BiPAP. El electrocardiograma solicitado muestra una taquicardia sinusal, sin signos de isquemia aguda. Se solicita una radiografía de tórax portátil que muestra infiltrado intersticial bilateral, con hilios prominentes y distribución vascular apical, compatible con congestión, además de visualizar cardiomegalia. Decide iniciar nitroglicerina por bomba de infusión continua. Reevalúa a la paciente y esta se encuentra vigil, se siente mejor, sus signos vitales son presión arterial (PA) 150/70 mmHg, frecuencia cardíaca (FC) 110 latidos por minuto, bien perfundida, saturación 94\% con frecuencia respiratoria (FR) 26 respiraciones por minuto, temperatura $36,2{ }^{\circ} \mathrm{C}, \mathrm{HGT} 120 \mathrm{mg} / \mathrm{dL}$. Llegan sus familiares le cuentan que la paciente tiene historia de 2 semanas 
de disnea progresiva, edema de extremidades inferiores, disnea paroxística nocturna, ortopnea y nicturia. Además, agregan que tiene mala adherencia a sus medicamentos y a dieta hiposódica. Niega aumento de la tos, fiebre, dolor torácico, viajes o cirugías recientes. Descarta uso de anticonceptivos u hormonas, síncope u otros síntomas asociados. No es alérgica a ningún medicamento, tampoco ha sido operada previamente y no tiene hospitalizaciones recientes. En este contexto usted se plantea como diagnóstico primario una edema pulmonar agudo (EPA) por una insuficiencia cardiaca descompensada secundaria a mala adherencia a tratamiento.

\section{Referencias}

Banzett R, O'Donnell C, Guilfoyle T, Parshall M, Schwartzstein R, Meek P, Gracely R \& Lansing R. (2015). Multidimensional Dyspnea Profile: an instrument for clinical and laboratory research. European Respiratory Journal 45, 1681-1691

Barbera A. \& Jones M. (2016). Dyspnea in the Elderly. Emergency Medicine Clinics of North America 34, 543-558.

Berliner D, Scheider N, Welte T \& Bauersachs J, (2016). The Differential Diagnosis of Dyspnea. Deutsches Ärzteblatt International 113, 834-845.

Braithwaite S \& Perina D. (2013). Dyspnea. Rosen's Emergency Medicine - Concepts and Clinical Practice. 8th Edition 25, 206-213.

Devos E. \& Jacobson L., (2016). Approach to adult patients with acute dysnea. Emergency Medicine Clinics of North America 34, 129-149.

Figarska S, Boezen H \& Vonk J. (2012). Dyspnea severity, changes in dyspnea status and mortality in the general population: the Vlagtwedde/Vlaardingen study. European Journal Epidemiology 27, 867-876.

Kelly AM, Keijzers G, Klim S, Graham CA, Craig S, Sen-Kuan W, Jones P, Holdgate A, Lawoko C. \& Laribi S. (2017). An Observational Study of Dyspnea in Emergency Departments:The Asia, Australia, and New Zealand Dyspnea in Emergency Departments Study (AANZDEM). Academic Emergency Medicine 24, 328-336.

Kline J, Jones JS. \& Colucciello SA. (1999). Dyspnea: Fear, Loathing, and Physiology. Emergency Medicine Practice 1, 3.

Kosowsky J. \& Kobayashi L. (2002). Acutely Decompensated Heart Failure: Diagnostic And Therapeutic Strategies For The New Millennium. Emergency Medicine Practice 4, 1-28.

Lam L, Cameron PA, Schneider HG, Abramson MJ, Müller C. \& Krum H. (2010). Meta-analysis: Effect of B-Type Natriuretic Peptide Testing on Clinical Outcomes in Patients With Acute Dyspnea in the Emergency Setting. Annals of Internal Medicine 153, 728-735.
Ministerio de Salud, (2008-2017). Departamento de Estadísticas e Información de Salud. Reportes de atenciones de urgencia por semana estadística. http://cognos.deis.cl/ibmcognos/cgi-bin/ cognos.cgi?b_action=cognosViewer\&ui.action=run\&ui.object $=/$ content/folder\%5B@name=\%27PUB\%27\%5D/folder\%5B@name=\%27REPORTES\%27\%5D/folder\%5B@name=\%27Atenciones\%20 de\%20Urgencia\%27\%5D/report\%5B@name=\%27Atenciones $\% 20$ Urgencia\%20-\%20Vista\%20por\%20semanas\%20-\%20Servicios\%27\%5D\&ui.name=Atenciones\%20Urgencia\%20-\%20Vista\%20por\%20 semanas\%20-\%20Servicios\&run.outputFormat=\&run.prompt=true el 16 de enero de 2020.

Mahler D. \& O' Donnell D. 2015. Recent advances in dyspnea. CHEST 147, 232 - 241.

Martino IF, Statti G, Fancoli F, Tinelli C. \& Bressan MA. (2014). Chest ultrasounds and $\mathrm{X}$-rays compared in patients with acute dyspnea in an Emergency Department. Critical Ultrasound Journal 6 (Suppl 2):A1

Meek P, Schwartzstein R, Adams L, Altrose M, Breslin E, Carrieri-Kohlman V, Gift A, Hanley M, Harvey A, Jones P, Killian K, Knebel A, Lareau S, Mahler D, O’Donnell D, Steele B, Stuhlbarg M. \& Titler M, (1999). Dyspnea: Mechanisms, assessment, and management: a consensus statement. American Journal of Respiratory Critical Care Medicine 159, 321-340.

Özkan B, Ünlüer E, Akyol P, Karagoz, Bayata M, Akoglu H, Oyar O, Dalli A \& Topal F. (2015). Stethoscope versus point-of-care ultrasound in the differential diagnosis of dyspnea: a randomized trial. European Journal of Emergency Medicine 22, 440-443.

Rochwerg B, Brochard L, Elliott M, Hess D, Hill NS, Nava S, Navalesi P, Antonelli M, Brozek J, Conti G, Ferrer M, Guntupalli K, Jaber S, Keenan S, Mancebo J, Mehta S. \& Raoof S. (2017). Official ERS/ ATS clinical practice guidelines: noninvasive ventilation for acute respiratory failure. The European Respiratory Journal 50, 1602426.

Seyedhosseini J, Bashizadeh-Fakhar G, Farzaneh S, Momeni M \& Karimialavijeh E. (2017). The impact of the BLUE protocol ultrasonography on the time taken to treat acute respiratory distress in the emergency department. American Journal of Emergency Medicine 35, 1815-1818.

Trinquart L, Ray P, Riou B. \& Teixeira A. (2011). Natriuretic peptide testing in EDs for managing acute dyspnea: a meta-analysis. American Journal of Emergency Medicine 29, 757-767

Viniol A, Beidatsch D, Frese T, Bergmann M, Grevenrath P, Schmidt L, Schwarm S, Haasenritter J, Bösner S, Becker A, (2015). Studies of the symptom dyspnea: A systematic review. BMC Family Practice 16, 152. 


\section{Sandoval et al.}

Wells P, Anderson D, Rodger M, Stiell I, Dreyer J, Barnes D, Forgie M, Kovacs G, Ward J. \& Kovacs M. (2001). Excluding pulmonary embolism at the bedside without diagnostic imaging: Management of patients with suspected pulmonary embolism presenting to the emergency department by using a simple clinical model and D-Dimer. Annals of Internal Medicine 135, 98-107.

Wimalasena Y, Kocierz L, Strong D, Watterson J. \& Burns B. (2017). Lung ultrasound: a useful tool in the assessment of the dyspnoeic patient in the emergency department. Fact or fiction?. Emergency Medicine Journal 35, 258-266
Zanobetti M, Scorpiniti M, Giglic C, Nazerian P, Vanni S, Innocenti F, Stefanone VT, Savinelli C, Coppa A, Bigiarini S, Cladi F, Tassinari I, Conti A, Grifoni S. \& Pini R. (2017). Point-of-Care Ultrasonography for Evaluation of Acute Dyspnea in the ED. CHEST 151, 1295-1301. 